PROCEEDINGS OF THE

AMERICAN MATHEMATICAL SOCIETY

Volume 131, Number 11, Pages 3431-3440

S 0002-9939(03)06929-6

Article electronically published on February 6, 2003

\title{
FRACTALS AND DISTRIBUTIONS ON THE $N$-TORUS
}

\author{
VICTOR L. SHAPIRO
}

(Communicated by Andreas Seeger)

\begin{abstract}
This paper establishes non-Cartesian product sets, called fractal carpets and fractal foam, as sets of uniqueness for a class of trigonometric series.
\end{abstract}

\section{Introduction}

Operating in $\mathbf{R}^{N}, N \geqq 2$, with $(x, y)=x_{1} y_{1}+\cdots+x_{N} y_{N},|x|=(x, x)^{1 / 2}$, and $\alpha x+\beta y=\left(\alpha x_{1}+\beta y_{1}, \ldots, \alpha x_{N}+\beta y_{N}\right)$, we call

$$
T_{N}=\left\{x=\left(x_{1}, \ldots, x_{N}\right): 0 \leqq x_{j}<1, j=1, \ldots, N\right\}
$$

the $N$-torus. In the sequel, working mod 1 in each variable, we shall describe fractal sets on $T_{N}$, called carpets in two dimensions and fractal foam in three dimensions (see [M, p. 133]), which will be sets of uniqueness for a class of distributions on the $N$-torus. The results to be presented here constitute an extension of our previous work, [Sh1], but now, unlike before, we show that the sets of uniqueness can be non-Cartesian product sets. We illustrate this fact with two examples.

Sh1] was motivated by the two-dimensional results in [Sh2 and analogous onedimensional results to be found in [Z1], [Z2], [KS], and [Sa].

We employ the notion of distributions as defined in [BJS, p. 168]. In particular, a distribution $S$ on $T_{N}$, also called a periodic distribution, is a real linear functional on $\mathcal{D}\left(T_{N}\right)$, the class of real functions in $\mathrm{C}^{(\infty)}\left(\mathbf{R}^{N}\right)$ which are periodic of period one in each variable, with the property that

(1.1) if $\phi_{k} \in \mathcal{D}\left(T_{N}\right)$ and $\left\|\phi_{k}\right\|_{t} \rightarrow 0$ as $k \rightarrow \infty$ for every $t>0$, then $S\left(\phi_{k}\right) \rightarrow 0$,

where $\left\|\phi_{k}\right\|_{t}$ is defined in (1.5) below. We will denote the class of such $S$ by $\mathcal{D}^{\prime}\left(T_{N}\right)$.

For $S \in \mathcal{D}^{\prime}\left(T_{N}\right)$, we set

$$
S^{\wedge}(m)=S[\cos 2 \pi(m, x)]-i S[\sin 2 \pi(m, x)]
$$

for every integral lattice point $m$ and observe from [BJS, p. 168] that there exists a positive integer $k$ such that

$$
\sum_{m}\left|S^{\wedge}(m)\right|(|m|+1)^{-k}<\infty .
$$

Received by the editors July 3, 2001 and, in revised form, May 25, 2002.

2000 Mathematics Subject Classification. Primary 42B35, 46F99; Secondary 42B05, 05A18.

Key words and phrases. Fractal, carpet, distribution, $N$-torus. 
Also, we have from this last-named reference that for $\phi \in \mathcal{D}\left(T_{N}\right)$,

$$
S(\phi)=\sum_{m} S^{\wedge}(m) \phi^{\wedge}(-m),
$$

where

$$
\phi^{\wedge}(m)=\int_{T_{N}} e^{-2 \pi i(m, x)} \phi(x) d x .
$$

Corresponding to [BJS, p. 166], we also have for $t$ an integer

$$
\|\phi\|_{t}^{2}=\sum_{m}\left[1+(2 \pi)^{2}|m|^{2}\right]^{t}\left|\phi^{\wedge}(m)\right|^{2} .
$$

In this paper, we deal with a subclass of $\mathcal{D}^{\prime}\left(T_{N}\right)$, namely the following:

$$
\mathcal{B}\left(T_{N}\right)=\left\{S \in \mathcal{D}^{\prime}\left(T_{N}\right): \lim _{|m| \rightarrow \infty}\left|S^{\wedge}(m)\right|=0\right\} .
$$

Next, we observe that the set $E \subset T_{N}$ is closed in the torus topology if and only if the set $E^{*}$ is a closed set in $\mathbf{R}^{N}$ where $E^{*}=\bigcup_{m}\{E+m\}$. If $\phi \in \mathcal{D}\left(T_{N}\right)$, we designate the set $\operatorname{supp}(\phi)$, called the support of $\phi$, by the following:

$$
\operatorname{supp}(\phi)=\left\{x \in T_{N}: \phi(x) \neq 0\right\}^{\sim}
$$

where $\sim$ denotes the closure in the torus topology. Also, given $G \subset T_{N}$ open in the torus topology and $S \in \mathcal{D}^{\prime}\left(T_{N}\right)$, we define $S=0$ in $G$ to mean

$$
S(\phi)=0 \quad \forall \phi \in \mathcal{D}\left(T_{N}\right) \text { such that } \operatorname{supp}(\phi) \subset G .
$$

A set $E \subset T_{N}$ closed in the torus topology will be called a $\mathcal{V}$-set if the following holds:

(i) $\exists\left\{p^{k}\right\}_{k=1}^{\infty}$, a sequence of integral lattice points, with $p^{k}=\left(p_{1}^{k}, \ldots, p_{N}^{k}\right)$ where $p_{j}^{k}$ is a positive integer for $j=1, \ldots, N$, and also

$$
\lim _{k \rightarrow \infty} p_{j}^{k}=\infty \text { for } j=1, \ldots, N
$$

(ii) $x \in E \Rightarrow\left(p_{1}^{k} x_{1}, \ldots, p_{N}^{k} x_{N}\right) \in E \bmod 1$ in each variable $\forall k$.

A set $Z \subset T_{N}$ which is of $N$-dimensional Lebesgue measure zero and also closed in the torus topology will be called a set of uniqueness for the class $\mathcal{B}\left(T_{N}\right)$, defined in (1.6), if the following holds:

$$
S \in \mathcal{B}\left(T_{N}\right) \text { and } S=0 \text { in } T_{N} \backslash Z \Rightarrow S \equiv 0 .
$$

We shall prove the following theorem.

Theorem. Suppose $Z \subset T_{N}$ is a closed set in the torus topology and also of $N$ dimensional Lebesgue measure zero. Suppose furthermore that $Z$ is a $\mathcal{V}$-set. Then $Z$ is a set of uniqueness for the class $\mathcal{B}\left(T_{N}\right)$.

\section{Fundamental lemmas}

If $\lambda \in \mathcal{D}\left(T_{N}\right)$ and $S \in \mathcal{D}^{\prime}\left(T_{N}\right)$, then we define $\lambda S \in \mathcal{D}^{\prime}\left(T_{N}\right)$ as follows:

$$
\lambda S(\phi)=S(\lambda \phi) \quad \forall \phi \in \mathcal{D}\left(T_{N}\right) .
$$

It is easy to check that this definition is all right because it meets the condition set forth in (1.1). 
Let $m$ be a fixed integral lattice point in $\mathbf{R}^{N}$. Then if $\lambda \in \mathcal{D}\left(T_{N}\right)$, it follows from (1.4) that

$$
\lambda e^{-2 \pi i(m, x)}=\sum_{p} \lambda^{\wedge}(p) e^{-2 \pi i(m-p, x)},
$$

and consequently from (1.2), (1.3), and (2.1) that for $S \in \mathcal{D}^{\prime}\left(T_{N}\right)$,

$$
(\lambda S)^{\wedge}(m)=\sum_{p} \lambda^{\wedge}(p) S^{\wedge}(m-p) .
$$

The first lemma that we establish is the following:

Lemma 1. Let $S \in D^{\prime}\left(T_{N}\right), \lambda \in D\left(T_{N}\right)$, and $G \subset T_{N}$ be open in the torus topology. Suppose $S=0$ in $G$, and $\lambda$ has its support in $G$. Then the distribution $\lambda S$ is such that $\lambda S \equiv 0$.

To prove the lemma, let $\phi \in \mathcal{D}\left(T_{N}\right)$. Then $\lambda \phi$ has its support in $G$. Consequently, it follows from (2.1) and the hypothesis of the lemma that $\lambda S(\phi)=0$, which establishes the lemma.

Lemma 2. Let $Z \subset T_{N}$ be a set closed in the torus topology and of $N$-dimensional Lebesgue measure zero. Suppose that

(1) $S \in \mathcal{B}\left(T_{N}\right)$ and that $S=0$ in $T_{N} \backslash Z$;

(2) $\exists\left\{\lambda_{k}\right\}_{k=1}^{\infty}$ with the following properties: (i) $\lambda_{k} \in \mathcal{D}\left(T_{N}\right) \forall k$, (ii) supp $\left(\lambda_{k}\right)$ $\subset T_{N} \backslash Z \quad \forall k$, (iii) $\exists C>0$ such that $\sum_{m}\left|\lambda_{k}^{\wedge}(m)\right| \leqq C \quad \forall k$, (iv) $\lim _{k \rightarrow \infty}\left|\lambda_{k}^{\wedge}(m)\right|$ $=0 \quad \forall m \neq 0$, and $(\mathrm{v}) \lim _{k \rightarrow \infty} \lambda_{k}^{\wedge}(0)=\alpha_{0} \neq 0$.

Then $S \equiv 0$.

To prove the lemma, we observe from (ii) that for each $k$ the support of $\lambda_{k}$ is in the open set $T_{N} \backslash Z$. Furthermore, $S=0$ in $T_{N} \backslash Z$. Hence, it follows from Lemma 1 that

$$
\lambda_{k} S \equiv 0 \quad \forall k
$$

Consequently, we obtain from (2.2) that

$$
0=\sum_{p} \lambda_{k}^{\wedge}(p) S^{\wedge}(m-p)
$$

for a fixed integral lattice point $m$. But then we have that

$$
-\lambda_{k}^{\wedge}(0) S^{\wedge}(m)=\sum_{p \neq 0} \lambda_{k}^{\wedge}(p) S^{\wedge}(m-p) .
$$

Since $m$ is a fixed integral lattice point, given $\varepsilon>0$, we have from (1.6) that there exists $R_{o}>1$ such that $\left|S^{\wedge}(m-p)\right|<\varepsilon$ for $|p| \geqq R_{o}$. From (iii) in the lemma and from (2.3), we then infer that

$$
\left|\lambda_{k}^{\wedge}(0) S^{\wedge}(m)\right|<\sum_{1 \leqq|p|<R_{o}}\left|\lambda_{k}^{\wedge}(p) S^{\wedge}(m-p)\right|+\varepsilon C .
$$

Passing to the limit as $\varepsilon \rightarrow 0$ on both sides of this last inequality and simultaneously making use of (iv) and (v) of the lemma gives us that

$$
\left|\alpha_{0} S^{\wedge}(m)\right| \leqq \varepsilon C .
$$

But $\varepsilon$ is an arbitrary positive number and $\alpha_{0} \neq 0$; so we conclude that $S^{\wedge}(m)=0$ for every lattice point $m$. It then follows from (1.3) that $S(\phi)=0 \forall \phi \in \mathcal{D}\left(T_{N}\right)$. Therefore, $S \equiv 0$, and the proof of the lemma is complete. 


\section{Proof of the Theorem}

To prove the Theorem, given that $Z \subset T_{N}$ is a closed set in the torus topology which is of $N$-dimensional Lebesgue measure zero and which is also a $\mathcal{V}$-set, we shall show the existence of a sequence $\left\{\lambda_{k}\right\}_{k=1}^{\infty}$ which meets the conditions $2(\mathrm{i})-2(\mathrm{v})$ in the hypothesis of Lemma 2. The Theorem will then follow from definition (1.10) and Lemma 2.

It is clear from the assumptions concerning $Z$ that there is an $x_{0} \in T_{N}$ and open ball with center $x_{0}$ and radius $r_{0}>0$, which we call $B\left(x_{0}, r_{0}\right)$, such that $Z \cap B\left(x_{0}, r_{0}\right)=\emptyset$. Hence, there is

$$
0<a_{j}^{1}<a_{j}^{2}<a_{j}^{3}<b_{j}^{3}<b_{j}^{2}<b_{j}^{1}<1, j=1, \ldots, N,
$$

with

$$
Q^{l}=\left[a_{1}^{l}, b_{1}^{l}\right] \times \cdots \times\left[a_{N}^{l}, b_{N}^{l}\right], \quad l=1,2,3,
$$

such that $Z \cap Q^{1}=\emptyset$. Since $Q^{3} \subset Q^{2}$ are rectangular parallelopipeds with the conditions in (3.1) holding, it is well known that there is a function $\lambda(x) \in \mathcal{D}\left(T_{N}\right)$ with the follow in $g$ properties:

(i) $\lambda(x)=1$ for $x \in Q^{3}$;

(ii) $\lambda(x)=0$ for $x \in T_{N} \backslash Q^{2}$, i.e., $\operatorname{supp}(\lambda) \subset Q^{2}$;

(iii) $\lambda(x) \geqq 0$ for $x \in T_{N}$.

To obtain the sequence $\left\{\lambda_{k}\right\}_{k=1}^{\infty}$ alluded to in the first paragraph above, we let $\left\{p^{k}\right\}_{k=1}^{\infty}$ be the sequence of integral lattice points introduced in (1.9)(i), (ii) used in the definition of $Z$ being a $\mathcal{V}$-set, and we define

$$
\lambda_{k}(x)=\lambda\left(p_{1}^{k} x_{1}, \ldots, p_{N}^{k} x_{N}\right) \quad \forall k \text { and } \forall x \in \mathbf{R}^{N} .
$$

Since $p_{j}^{k}$ is a positive integer for $j=1, \ldots, N$, and since $\lambda \in \mathcal{D}\left(T_{N}\right)$, it follows from (3.3) that $\lambda_{k} \in \mathcal{D}\left(T_{N}\right)$. Hence, (2)(i) in Lemma 2 for our sequence $\left\{\lambda_{k}\right\}$ is established.

To establish (2)(ii) in Lemma 2, we observe that both $Q^{1}$ and $Z$ are compact subsets of $T_{N}$ in the torus topology and $Z \cap Q^{1}=\emptyset$. Consequently, $\exists \varepsilon_{0}>0$ such that

$$
B\left(x, \varepsilon_{0}\right) \cap Q^{1 *}=\emptyset \text { for } x \in Z^{*}
$$

where $Q^{1 *}=\bigcup_{m}\left\{Q^{1}+m\right\}$ and $Z^{*}=\bigcup_{m}\{Z+m\}$. Now for $k$ fixed, let

$$
\left|p^{k}\right|^{2}=\sum_{j=1}^{N}\left|p_{j}^{k}\right|^{2} .
$$

Then it follows from (3.4) with $\varepsilon_{1}=\varepsilon_{0} /\left|p^{k}\right|$ that

$$
x+\left(p_{1}^{k} y_{1}, \ldots, p_{N}^{k} y_{N}\right) \notin Q^{1 *} \quad \text { for }|y|<\varepsilon_{1} \text { and } x \in Z^{*} .
$$

By $(1.9)(\mathrm{ii}), x=\left(x_{1}, \ldots, x_{N}\right) \in Z \Longrightarrow\left(p_{1}^{k} x_{1}, \ldots, p_{N}^{k} x_{N}\right) \in Z^{*}$. Therefore if $x \in Z$ and $|y|<\varepsilon_{1}$,

$$
\left(p_{1}^{k} x_{1}, \ldots, p_{N}^{k} x_{N}\right)+\left(p_{1}^{k} y_{1}, \ldots, p_{N}^{k} y_{N}\right) \notin Q^{1 *}
$$

From (3.1) and (3.2), we see that $Q^{2 *} \subset Q^{1 *}$ and from (ii) in the properties of $\lambda(x)$ that supp $(\lambda) \subset Q^{2}$. Consequently, it follows from (3.3) and (3.5) that if $x \in Z$ and $|y|<\varepsilon_{1}, \lambda_{k}(x+y)=0$. Hence, $Z \cap \operatorname{supp}\left(\lambda_{k}\right)=\emptyset$, and (2)(ii) of Lemma 2 is established. 
To establish (2)(iii) in Lemma 2, we observe that $\lambda(x) \in \mathcal{D}\left(T_{N}\right)$. Consequently,

$$
\lambda(x)=\sum_{m} \lambda^{\wedge}(m) e^{2 \pi i(m, x)}
$$

where

$$
\sum_{m}\left|\lambda^{\wedge}(m)\right|=C<\infty
$$

It follows therefore from (3.3) that

$$
\lambda_{k}(x)=\lambda^{\wedge}(0)+\sum_{m \neq 0} \lambda^{\wedge}(m) e^{2 \pi i\left(p_{1}^{k} m_{1} x_{1}+\cdots+p_{N}^{k} m_{N} x_{N}\right)}
$$

for $x \in T_{N}$. Hence,

$$
\sum_{m}\left|\lambda_{k}^{\wedge}(m)\right|=\sum_{m}\left|\lambda^{\wedge}(m)\right|=C<\infty,
$$

which fact establishes (2)(iii) in Lemma 2.

To establish (2)(iv) in Lemma 2, let $m_{0}$ be an arbitrary but fixed integral lattice with $m_{0} \neq 0$. It follows from (1.9) that $\exists k_{0}>0$ such that for $k>k_{0}$

$$
\left(\sum_{j=1}^{N}\left|p_{j}^{k} m_{j}\right|^{2}\right)^{1 / 2} \geqq \min \left(p_{1}^{k}, \ldots, p_{N}^{k}\right) \geqq\left|m_{0}\right|+1
$$

for all $m \neq 0$. Consequently, it follows from (3.8) that for $k>k_{0}$,

$$
\lambda_{k}^{\wedge}\left(m_{0}\right)=0 \text {, }
$$

and (2)(iv) in Lemma 2 is established.

Next, we use (3.8) once again and obtain that

$$
\lambda_{k}{ }^{\wedge}(0)=\lambda^{\wedge}(0) \quad \forall k \text {. }
$$

From the defining properties (i), (ii), (iii) of $\lambda(x)$ stated above, we see that

$$
0<\int_{T_{N}} \lambda(x)=\lambda^{\wedge}(0) .
$$

We conclude from these last two facts that indeed $\lim _{k \rightarrow \infty} \lambda_{k}{ }^{\wedge}(0)=\alpha_{0} \neq 0$. Hence $(2)(\mathrm{v})$ in Lemma 2 is established, and the proof of the theorem is complete.

\section{EXAmples of SETS OF UNiQUeness}

In order to show that a set $Z \subset T_{N}$ is a set of uniqueness for the class $\mathcal{B}\left(T_{N}\right)$, according to the theorem, we need only show that (i) it is closed in the torus sense, (ii) it is of $N$-dimensional Lebesgue measure zero, and (iii) it is a $\mathcal{V}$-set. We shall do this for two different examples; the first will take place in dimension $N=3$ and the second in dimension $N=2$. Each example will constitute a non-Cartesian product set. It will also be clear that both examples hold for $N \geqq 3$, but the notation in

the higher dimensional cases is considerably more cumbersome. Also, example 2 covers example 1 in dimension $N=2$.

For $N=3$, the set we will deal with is alluded to in Mandelbrot's book as triadic fractal foam [M, p. 133], and we will refer to it as $T F F$. We will define $T F F$ in $\bar{T}_{3}$ where

$$
\bar{T}_{3}=\left\{x=\left(x_{1}, x_{2}, x_{3}\right): 0 \leqq x_{j} \leqq 1, j=1,2,3\right\} .
$$


Our set of uniqueness $Z$ will then be

$$
Z=T F F \cap T_{3}
$$

To define $T F F$, subdivide $\bar{T}_{3}$ into 27 closed congruent cubes by cutting $\bar{T}_{3}$ with planes parallel to the three axes, i.e., $x_{j}=1 / 3,2 / 3$ for $j=1,2,3$. Each cube has a distinguished point within it, namely $x^{j_{1}, 1}$ which is the point with smallest Euclidean norm in each cube. Each $x^{j_{1}, 1}$ corresponds to a unique triple

$$
x^{j_{1}, 1} \longleftrightarrow\left(\varepsilon_{1}, \delta_{1}, \zeta_{1}\right)
$$

with $x^{j_{1}, 1}=\left(\varepsilon_{1} / 3, \delta_{1} / 3, \zeta_{1} / 3\right)$ where $\varepsilon_{1}, \delta_{1}, \zeta_{1}$ run through the numbers $0,1,2$ with one caveat: we do not allow the triple with $\varepsilon_{1}=\delta_{1}=\zeta_{1}=1$ since we are going to remove the open cube corresponding to this point. We shall define an ordering on different triples of the nature $\left(\varepsilon_{1}, \delta_{1}, \zeta_{1}\right) \neq\left(\varepsilon_{1}^{\prime}, \delta_{1}^{\prime}, \zeta_{1}^{\prime}\right)$ as follows:

$$
\left(\varepsilon_{1}, \delta_{1}, \zeta_{1}\right) \prec\left(\varepsilon_{1}^{\prime}, \delta_{1}^{\prime}, \zeta_{1}^{\prime}\right) \text { means }
$$

(i) $\varepsilon_{1}<\varepsilon_{1}^{\prime}$ or (ii) $\varepsilon_{1}=\varepsilon_{1}^{\prime}$ and $\delta_{1}<\delta_{1}^{\prime}$ or (iii) $\varepsilon_{1}=\varepsilon_{1}^{\prime}$ and $\delta_{1}=\delta_{1}^{\prime}$ and $\zeta_{1}<\zeta_{1}^{\prime}$. This also imposes an ordering on $\left\{x^{j_{1}, 1}\right\}$ via (4.2).

Now we have 26 triples, and we count them out according to this $\prec$-ordering, giving us $\left\{x^{j_{1}, 1}\right\}_{j_{1}=1}^{26}$. Thus $x^{1,1}=(0,0,0), x^{2,1}=(0,0,1 / 3), x^{3,1}=(0,0,2 / 3)$, $x^{4,1}=(0,1 / 3,0), \ldots, x^{26,1}=(2 / 3,2 / 3,2 / 3)$. The closed cube which has $x^{j_{1}, 1}$ as its distinguished point, we label $I^{j_{1}, 1}$. We then define $I^{1} \subset \bar{T}_{3}$ to be the closed set

$$
I^{1}=\bigcup_{j_{1}=1}^{26} I^{j_{1}, 1} .
$$

In each of the 26 cubes, which have sides of length $1 / 3$, we now perform the same operation as above, obtaining $(26)^{2}$ cubes, which now have sides of length $(1 / 3)^{2}$. Each of these last-mentioned cubes has a distinguished point

$$
x^{j_{2}, 2}=x^{j_{1}, 1}+\left(\varepsilon_{2} / 3^{2}, \delta_{2} / 3^{2}, \zeta_{2} / 3^{2}\right)
$$

where $\varepsilon_{2}, \delta_{2}, \zeta_{2}$ run through the numbers $0,1,2$, and we do not allow the triple with $\varepsilon_{2}=\delta_{2}=\zeta_{2}=1$. These triples have an ordering imposed on them by (4.3) which in turn gives an ordering on $\left\{x^{j_{2}, 2}\right\}$ defined as follows:

$$
x^{j_{2}, 2} \prec x^{j_{2}^{\prime}, 2} \text { means }
$$

$$
\text { (i) } x^{j_{1}, 1} \prec x^{j_{1}^{\prime}, 1} \text { or (ii) } x^{j_{1}, 1}=x^{j_{1}^{\prime}, 1} \text { and }\left(\varepsilon_{2}, \delta_{2}, \zeta_{2}\right) \prec\left(\varepsilon_{2}^{\prime}, \delta_{2}^{\prime}, \zeta_{2}^{\prime}\right) \text {. }
$$

We then count out the $(26)^{2}$ points according to this ordering and obtain $\left\{x^{j_{2}, 2}\right\}_{j_{2}=1}^{(26)^{2}}$. The closed cube containing $x^{j_{2}, 2}$ as its distinguished point we call $I^{j_{2}, 2}$. We then define $I^{2} \subset I^{1} \subset \bar{T}_{3}$ to be the closed set

$$
I^{2}=\bigcup_{j_{2}=1}^{(26)^{2}} I^{j_{2}, 2} .
$$

In each of the $(26)^{2}$ cubes which have sides of length $(1 / 3)^{2}$, we now perform the same operation as before obtaining $(26)^{3}$ cubes with each having sides of length $(1 / 3)^{3}$. We get distinguished points in each of these cubes and put an ordering on them similar to the procedure in (4.4) to obtain $\left\{x^{j_{3}, 3}\right\}_{j_{3}=1}^{(26)^{3}}$. Next, in a procedure similar to (4.5), we get the closed set $I^{3}$ with $I^{3} \subset I^{2} \subset I^{1} \subset \bar{T}_{3}$. 
Continuing in this manner, we get the decreasing sequence of closed sets $\left\{I^{n}\right\}_{n=1}^{\infty}$ with $I^{n+1} \subset I^{n} \subset \bar{T}_{3}$ where each $I^{n}$ consists of $(26)^{n}$ cubes each with sides of length $(1 / 3)^{n}$. The set $T F F$ is then defined to be

$$
T F F=\bigcap_{n=1}^{\infty} I^{n}
$$

With $Z$ defined by (4.1) where $T F F$ is defined by (4.6), we see that $Z$ is closed in the torus sense because every point in the boundary of $\bar{T}_{3}$ is contained in $T F F$. Also since the Lebesgue measure of each $I^{n}$ in $(4.6)$ is $(26 / 27)^{n}$, we see that $T F F$ is of $N$-dimensional Lebesgue measure zero; hence by (4.1) the same can be said of $Z$. Consequently, we conclude from the conditions in the hypothesis of the theorem to show that $Z$ is a set of uniqueness for the class $\mathcal{B}\left(T_{3}\right)$; it only remains to show that $Z$ is a $\mathcal{V}$-set according to the definition given in (1.9). We claim

$$
x \in Z \Rightarrow\left(3^{k} x_{1}, 3^{k} x_{2}, 3^{k} x_{3}\right) \in Z \bmod 1 \text { in each variable }
$$

for $k$ a positive integer where $x=\left(x_{1}, x_{2}, x_{3}\right)$. Once (4.7) is established, it then follows from (1.9) that $Z$ is indeed a $\mathcal{V}$-set. To show that (4.7) holds, it is clearly sufficient to show that it holds in the special case when $k=1$, i.e.,

$$
x \in Z \Rightarrow\left(3 x_{1}, 3 x_{2}, 3 x_{3}\right) \in Z \quad \bmod 1 \text { in each variable. }
$$

It follows from the definition of $T F F$ in (4.6) that given $x_{o} \in T F F, \exists\left\{x_{o}^{j_{n}, n}\right\}_{n=1}^{\infty}$ where each $x_{o}^{j_{n}, n}$ is a distinguished point of one of the $(26)^{n}$ cubes in $I^{n}$ of sides $(1 / 3)^{n}$ such that

$$
\left\|x_{o}^{j_{n}, n}-x_{o}\right\| \rightarrow 0 \text { as } n \rightarrow \infty .
$$

Consequently, to show that (4.8) holds it is sufficient to show that it holds when $x$ is a distinguished point $x^{j_{n}, n}$.

If $x=x^{j_{1}, 1}$, then it follows from the enumeration of the 26 such points given below (4.3) that the conclusion in (4.8) holds. Hence from the above discussion $Z$ will be a $\mathcal{V}$-set, if we show the following:

Given $x^{j_{n}, n}=\left(x_{1}^{j_{n}, n}, x_{2}^{j_{n}, n}, x_{3}^{j_{n}, n}\right)$ a distinguished point in an $I^{j_{n}, n}$, then

$$
\left(3 x_{1}^{j_{n}, n}, 3 x_{2}^{j_{n}, n}, 3 x_{3}^{j_{n}, n}\right)=x^{j_{n-1}, n-1} \bmod 1 \text { in each variable }
$$

for $n \geqq 2$ where $x^{j_{n-1}, n-1}$ is a distinguished point in an $I^{j_{n-1}, n-1}$.

It is clear from the representation of $x^{j_{2}, 2}$ given above (4.4) that

$$
x^{j_{2}, 2}=\left(\frac{\varepsilon_{1}}{3}+\frac{\varepsilon_{2}}{3^{2}}, \frac{\delta_{1}}{3}+\frac{\delta_{2}}{3^{2}}, \frac{\zeta_{1}}{3}+\frac{\zeta_{2}}{3^{2}}\right)
$$

where $\varepsilon_{i}, \delta_{i}, \zeta_{i}$ run through the numbers $0,1,2$, and we do not allow $\varepsilon_{i}=\delta_{i}=\zeta_{i}=1$ for $i=1,2$. Exactly similar reasoning shows that

$$
x^{j_{n}, n}=\left(\sum_{i=1}^{n} \frac{\varepsilon_{i}}{3^{i}}, \sum_{i=1}^{n} \frac{\delta_{i}}{3^{i}}, \sum_{i=1}^{n} \frac{\zeta_{i}}{3^{i}}\right)
$$

where now $\varepsilon_{i}=\delta_{i}=\zeta_{i}=1$ is not allowed for $i=1, \ldots, n$. From (4.10), we see that

$$
\left(3 x_{1}^{j_{n}, n}, 3 x_{2}^{j_{n}, n}, 3 x_{3}^{j_{n}, n}\right)=\left(\varepsilon_{1}+\sum_{i=1}^{n-1} \frac{\varepsilon_{i+1}}{3^{i}}, \delta_{1}+\sum_{i=1}^{n-1} \frac{\delta_{i+1}}{3^{i}}, \zeta_{1}+\sum_{i=1}^{n-1} \frac{\zeta_{i+1}}{3^{i}}\right) .
$$

But $\varepsilon_{1}, \delta_{1}$, and $\zeta_{1}$ are each non-negative integers, and we conclude from this last equality that (4.9) does indeed hold. Hence $Z$ defined by (4.1) is a $\mathcal{V}$-set, and our example is complete. 
Our next example will take place in dimension $N=2$. We will call it a generalized carpet and refer to it as $G C_{p q}$ where $p$ and $q$ are both positive integers strictly greater than 2. The set $G C_{p q}$ will be a subset of $\bar{T}_{2}$ where

$$
\bar{T}_{2}=\left\{x=\left(x_{1}, x_{2}\right): 0 \leqq x_{j} \leqq 1, j=1,2\right\} .
$$

In particular, when $p=q=3, G C_{p q}$ will be the set referred to in the literature as the Sierpinski carpet [M, p. 144].

To define $G C_{p q}$, subdivide $\bar{T}_{2}$ into $p q$ closed congruent rectangles by cutting $\bar{T}_{2}$ with lines parallel to the two axes, i.e., $x_{1}=1 / p, 2 / p, \ldots,(p-1) / p, x_{2}=1 / q, 2 / q, \ldots$, $(q-1) / q$. Each rectangle has a distinguished point within it, namely $x^{j_{1}, 1}$ which is the point with smallest Euclidean norm in each rectangle. Each $x^{j_{1}, 1}$ corresponds to a unique double

$$
x^{j_{1}, 1} \longleftrightarrow\left(\varepsilon_{1}, \delta_{1}\right)
$$

with $x^{j_{1}, 1}=\left(\varepsilon_{1} / p, \delta_{1} / q\right)$ where $\varepsilon_{1}$ and $\delta_{1}$ run through the numbers $0,1, \ldots$, $p-1$ and $0,1, \ldots, q-1$, respectively. There is a caveat however; the doubles with $\varepsilon_{1}=1, \ldots, p-2$, and simultaneously $\delta_{1}=1, \ldots, q-2$, are not allowed, for the rectangles corresponding to these points will be removed, i.e., the middle $(p-2)(q-2)$ rectangles will be deleted. An ordering on different doubles of the nature $\left(\varepsilon_{1}, \delta_{1}\right) \neq\left(\varepsilon_{1}^{\prime}, \delta_{1}^{\prime}\right)$ is then defined as follows:

$$
\left(\varepsilon_{1}, \delta_{1}\right) \prec\left(\varepsilon_{1}^{\prime}, \delta_{1}^{\prime}\right) \text { means }
$$

(i) $\varepsilon_{1}<\varepsilon_{1}^{\prime}$ or (ii) $\varepsilon_{1}=\varepsilon_{1}^{\prime}$ and $\delta_{1}<\delta_{1}^{\prime}$. This also imposes an ordering on $\left\{x^{j_{1}, 1}\right\}_{j_{1}=1}^{\gamma}$ via (4.11) where $\gamma$ is the integer

$$
\gamma=p q-(p-2)(q-2) .
$$

In particular, we see that $x^{1,1}=(0,0), x^{2,1}=(0,1 / q), x^{3,1}=(0,2 / q), \ldots, x^{\gamma, 1}=$ $((p-1) / p,(q-1) / q)$. We also observe that $x^{q, 1}=(0,(q-1) / q), x^{q+1,1}=(1 / p, 0)$, and $x^{q+2,1}=(1 / p,(q-1) / q)$. The closed rectangle which has $x^{j_{1}, 1}$ as its distinguished point we label $I^{j_{1}, 1}$. We then define $I^{1} \subset \bar{T}_{2}$ to be the closed set

$$
I^{1}=\bigcup_{j_{1}=1}^{\gamma} I^{j_{1}, 1}
$$

In each of the $\gamma$ closed rectangles, which have sides of length $1 / p$ and $1 / q$, we now perform the same operation as above, obtaining $\gamma^{2}$ closed rectangles, which now have sides of length $(1 / p)^{2}$ and $(1 / q)^{2}$. Each of these last-mentioned rectangles has a distinguished point within it, namely $x^{j_{2}, 2}$, where

$$
x^{j_{2}, 2}=x^{j_{1}, 1}+\left(\varepsilon_{2} / p^{2}, \delta_{2} / q^{2}\right),
$$

and where $\varepsilon_{2}$ and $\delta_{2}$ run through the numbers $0,1, \ldots, p-1$ and $0,1, \ldots, q-1$, respectively. Also, we do not allow the doubles with $\varepsilon_{2}=1, \ldots, p-2$, and simultaneously $\delta_{2}=1, \ldots, q-2$. The doubles $\left(\varepsilon_{2}, \delta_{2}\right) \neq\left(\varepsilon_{2}^{\prime}, \delta_{2}^{\prime}\right)$ have an ordering imposed upon them by (4.12), which, in turn, imposes an ordering on the distinguished points given by $x^{j_{2}, 2} \prec x^{j_{2}^{\prime}, 2}$ akin to the ordering given in (4.4). We then count out the $\gamma^{2}$ points according to this ordering and obtain $\left\{x^{j_{2}, 2}\right\}_{j_{2}=1}^{\gamma^{2}}$. The closed rectangle of sides $(1 / p)^{2}$ and $(1 / q)^{2}$ containing $x^{j_{2}, 2}$ as its distinguished point we call $I^{j_{2}, 2}$. 
We then define $I^{2} \subset I^{1} \subset \bar{T}_{2}$ to be the closed set

$$
I^{2}=\bigcup_{j_{2}=1}^{\gamma^{2}} I^{j_{2}, 2} .
$$

Continuing in this manner, we get the decreasing sequence of closed sets $\left\{I^{n}\right\}_{n=1}^{\infty}$ with $I^{n+1} \subset I^{n} \subset \bar{T}_{2}$ where each $I^{n}$ consists of $\gamma^{n}$ rectangles each with sides of length $(1 / p)^{n}$ and $(1 / q)^{n}$. The set $G C_{p q}$ is then defined to be

$$
G C_{p q}=\bigcap_{n=1}^{\infty} I^{n}
$$

Next, we define $Z$ to be the set

$$
Z=G C_{p q} \cap T_{2}
$$

and observe that $Z$ is closed in the torus sense because every point in the boundary of $\bar{T}_{2}$ is contained in $G C_{p q}$. Also, since the Lebesgue measure of each $I^{n}$ is $(\gamma / p q)^{n}$ where $\gamma=p q-(p-2)(q-2)$, we see from (4.13) and (4.14) that the 2-dimensional Lebesgue measure of $Z$ is zero. Hence according to the conditions in the hypothesis of the theorem, to show that $Z$ is a set of uniqueness for the class $\mathcal{B}\left(T_{2}\right)$, it only remains to show that $Z$ is a $\mathcal{V}$-set, i.e., that the conditions set forth in (1.9) hold.

We claim

$$
x \in Z \Rightarrow\left(p^{k} x_{1}, q^{k} x_{2}\right) \in Z \quad \bmod 1 \text { in each variable }
$$

for $k$ a positive integer and with $x=\left(x_{1}, x_{2}\right)$. Once (4.15) is established, then it follows from (1.9) that $Z$ is indeed a $\mathcal{V}$-set. To show that (4.15) holds, it is clearly sufficient to show that it holds in the special case when $k=1$, i.e.,

$$
x \in Z \Rightarrow\left(p x_{1}, q x_{2}\right) \in Z \quad \bmod 1 \text { in each variable. }
$$

Using the same argument that we used after (4.8), we see that to show that $Z$ is a $\mathcal{V}$-set, we need only show that (4.16) holds for the special case when $x=x^{j_{n}, n}$, a distinguished point in one of the closed rectangles $I^{j_{n}, n}$ with sides $(1 / \mathrm{p})^{n}$ and $(1 / \mathrm{q})^{n}$.

If $x=x^{j_{1}, 1}$, then it follows from the enumeration of such points below (4.12) that (4.16) does indeed hold. Hence to show that $Z$ is a $\mathcal{V}$-set, it only remains to establish the fact that (4.16) holds when $x=x^{j_{n}, n}$ for $n \geqq 2$. This will be accomplished if we show that the following fact holds:

Given $x^{j_{n}, n}=\left(x_{1}^{j_{n}, n}, x_{2}^{j_{n}, n}\right)$ a distinguished point in an $I^{j_{n}, n}$, then

$$
\left(p x_{1}^{j_{n}, n}, q x_{2}^{j_{n}, n}\right)=x^{j_{n-1}, n-1} \bmod 1 \text { in each variable }
$$

for $n \geqq 2$ where $x^{j_{n-1}, n-1}$ is a distinguished point in an $I^{j_{n-1}, n-1}$.

It is clear from the representation of $x^{j_{2}, 2}$ given above that

$$
x^{j_{2}, 2}=\left(\frac{\varepsilon_{1}}{p}+\frac{\varepsilon_{2}}{p^{2}}, \frac{\delta_{1}}{q}+\frac{\delta_{2}}{q^{2}}\right)
$$

where $\varepsilon_{i}$ and $\delta_{i}$ run through the numbers $0, \ldots, p-1$, and $0, \ldots, q-1$, respectively, and we do not allow $\varepsilon_{i}=1, \ldots, p-2$ and simultaneously $\delta_{i}=1, \ldots, q-2$ for $i=1,2$. Exactly similar reasoning shows that

$$
x^{j_{n}, n}=\left(\sum_{i=1}^{n} \frac{\varepsilon_{i}}{p^{i}}, \sum_{i=1}^{n} \frac{\delta_{i}}{q^{i}}\right)
$$


where $\varepsilon_{i}$ and $\delta_{i}$ are exactly as before, now with $i=1, \ldots, n$. From (4.18), we see that

$$
\left(p x_{1}^{j_{n}, n}, q x_{2}^{j_{n}, n}\right)=\left(\varepsilon_{1}+\sum_{i=1}^{n-1} \frac{\varepsilon_{i+1}}{p^{i}}, \delta_{1}+\sum_{i=1}^{n-1} \frac{\delta_{i+1}}{q^{i}}\right) .
$$

But $\varepsilon_{i}$ and $\delta_{i}$ are each non-negative integers, and we conclude from this last equality and (4.18) that (4.17) does indeed hold. Hence, $Z$ defined by (4.14) is a $\mathcal{V}$-set, and our example is complete.

In closing, we point out that the $H^{J}$-sets, defined in Sh2] for dimension $N=2$ and in [AW] for dimensions $N \geqq 3$, can also be shown to be sets of uniqueness for the class $\mathcal{B}\left(T_{N}\right)$ with respect to distributions on the $N$-torus.

\section{REFERENCES}

[AW] J. M. Ash and G. Wang, Sets of uniqueness for spherically convergent multiple trigonometric series, preprint, 1999, 20 pages.

[BJS] L. Bers, F. John, and M. Schechter, Partial Differential Equations, Interscience Publishers, New York, 1964.

[KS] J. Kahane and R. Salem, Ensembles Parfait et Series Trigonométriques, Hermann, Paris, 1963. MR 28:3279

[M] B. B. Mandelbrot, The Fractal Geometry of Nature, W. H. Freeman and Company, New York, 1982. MR 84h:00021

[Sa] R. Salem, Algebraic Numbers and Fourier Analysis, Heath, Boston, 1963. MR 28:1169

[Sh1] V. L. Shapiro, Algebraic integers and distributions on the $N$-torus, J. Functional Analysis 13 (1973), pp. 138-153. MR 50:890

[Sh2] V. L. Shapiro, Sets of uniqueness on the 2-torus, Trans. Amer. Soc. 165 (1972), pp. 127-147. MR 46:7798

[Z1] A. Zygmund, Trigonometric Series, 2nd ed. Vol. I, Cambridge Univ. Press, New York, 1959. MR 21:6498

[Z2] A. Zygmund, Trigonometric Series, 2nd ed. Vol. II, Cambridge Univ. Press, New York, 1959. MR 21:6498

Department of Mathematics, University of California, Riverside, California 925210135

E-mail address: shapiro@math.ucr.edu 